\title{
MATERIAIS À BASE DE ÓXIDO DE FERRO PARA OXIDAÇÃO DE COMPOSTOS PRESENTES NO EFLUENTE DA DESPOLPA DO CAFÉ
}

\author{
Maraísa Gonçalves, Mário César Guerreiro*, Luiz Carlos Alves Oliveira e Cristian Luciana da Rocha \\ Departamento de Química, Universidade Federal de Lavras, CP 37, 37200-000 Lavras - MG, Brasil
}

Recebido em 3/7/07; aceito em 16/4/08; publicado na web em 26/9/08

\begin{abstract}
OXIDATION OF ORGANIC COMPOUNDS IN WASTEWATER FROM THE HUMID PROCESSING OF COFFEE BERRIES. Materials based on pure iron oxide and impregnated with niobia $\left(\mathrm{Nb}_{2} \mathrm{O}_{5}\right)$ were prepared. Their catalytic activities were tested on the oxidation of compounds present in the wastewater from the processing of coffee berries. Particularly caffeine and catechol were tested. The oxidation reactions were carried out with the following systems (i) $\mathrm{UV} / \mathrm{H}_{2} \mathrm{O}_{2}$, (ii) photo-Fenton and (iii) heterogeneous Fenton. All materials were characterized with X-ray diffraction, Mössbauer and infrared spectroscopy. Iron was mainly in the forms of goethite and maghemite. The oxidation kinetics were monitored by UV-vis and the oxidation products were monitored by mass spectrometry. The photo-Fenton reaction presented highest oxidation efficiency, removing $98 \%$ of all caffeine and catechol contents.
\end{abstract}

Keywords: oxidation; niobia; iron oxide.

\section{INTRODUÇÃO}

No Brasil, um dos produtos agrícolas geradores de grande carga poluidora é o café, não somente pela elevada escala de produção, mas também pela pequena fração ponderal do fruto efetivamente utilizada como produto final. Um café de boa qualidade é processado de forma que frutos sadios sejam separados dos defeituosos. Isso pode ser feito de, pelo menos, duas formas: por via seca, isto é, secando integralmente os frutos, com geração de proporção considerável de resíduos sólidos ou, por via úmida, que consiste na secagem dos grãos descascados e despolpados que, além do rejeito sólido, gera grande volume de efluente líquido.

O descascamento e o despolpamento úmido dos frutos do cafeeiro vêm crescendo nos últimos anos, na medida em que confere melhor qualidade ao produto e reduz o custo e o espaço de secagem. O volume de água utilizada nessa fase do processamento é enorme: estima-se que para despolpar mil litros de café-cereja sejam consumidos em média $2,5 \mathrm{~m}^{3}$ de água. No Brasil, aproximadamente $1,4 \%$ da produção de grãos é despolpada por via úmida, gerando cerca de 2,5 milhões $\mathrm{m}^{3}$ de efluente todos os anos.

Esse efluente tem elevada carga orgânica, com demanda química de oxigênio (DQO) de aproximadamente $7.000 \mathrm{mg} \mathrm{L}^{-1}$ e grande diversidade de compostos orgânicos, como cafeína, açúcares e compostos fenólicos, ${ }^{1}$ impróprios para descarte direto em solos ou cursos d'água. ${ }^{2}$ Dessa maneira, o desenvolvimento de sistemas eficazes na remoção da carga orgânica ou a transformação de compostos recalcitrantes em matéria orgânica biodegradável apresenta-se como alternativa viável para minimização do impacto ambiental causado.

A cafeína, um alcalóide pertencente ao grupo das metilxantinas, composto estimulante, muito persistente no meio devido à sua estabilidade e ter também alta solubilidade em água $\left(21,7 \mathrm{~g} \mathrm{~L}^{-1}\right)$, podendo ser utilizado como marcador da poluição da despolpa do café em cursos d água, pois se encontra em uma concentração ${ }^{1}$ de aproximadamente $35 \mathrm{mgL}^{-1}$. As leis ambientais não determinam a concentração de cafeína permitida para descarte de um efluente.

Os compostos fenólicos dos efluentes são altamente tóxicos aos microrganismos, mesmo em baixas concentrações, causando danos

\footnotetext{
*e-mail: guerrero@ufla.br
}

ao meio aquático, onde muitas vezes é descartado. No efluente da despolpa sua concentração foi de $90 \mathrm{mgL}^{-1}$, sendo 150 vezes superior à concentração máxima permitida pela legislação ambiental $(0,5$ $\mathrm{mgL}^{-1}$ ) para descarte de efluentes. ${ }^{1}$

Os processos oxidativos avançados têm sido investigados como promissores para degradação de grande variedade de compostos orgânicos. Baseiam-se na formação de radicais altamente oxidantes, como o radical hidroxila ou o hidroperóxido, que são capazes de transformar os contaminantes presentes no meio em moléculas menos poluentes, ou mesmo mineralizá-las. ${ }^{3-8}$

No presente trabalho, foi estudada a oxidação da cafeína e de compostos fenólicos representados pelo catecol. Ambas as classes de compostos encontram-se presentes no efluente da despolpa dos frutos do cafeeiro. Os processos de oxidação empregados foram Fenton, foto-Fenton e $\mathrm{UV} / \mathrm{H}_{2} \mathrm{O}_{2}$; os materiais testados como catalisadores foram óxido de ferro puro (Fe-puro) e compósito óxido de ferro disperso na superfície de nióbia 5/1 (Fe/Nb). A escolha do suporte utilizado neste trabalho, a nióbia, foi devida às suas propriedades catalíticas e sua grande disponibilidade no Brasil.

\section{PARTE EXPERIMENTAL}

\section{Preparação dos materiais}

O óxido de ferro (Fe-puro) foi preparado gotejando-se $30 \mathrm{~mL}$ de $\mathrm{NaOH}\left(5 \mathrm{~mol} \mathrm{~L}^{-1}\right)$ em um béquer contendo $\mathrm{FeCl}_{3} \cdot 6 \mathrm{H}_{2} \mathrm{O}(28 \mathrm{mmol})$, $\mathrm{FeSO}_{4} .7 \mathrm{H}_{2} \mathrm{O}(14 \mathrm{mmol})$ e $100 \mathrm{~mL}$ de $\mathrm{H}_{2} \mathrm{O}$ a $70{ }^{\circ} \mathrm{C}$. O compósito óxido de ferro disperso em nióbia $\left(\mathrm{Nb}_{2} \mathrm{O}_{5}\right)$, na proporção $5 / 1(\mathrm{Fe} / \mathrm{Nb})$, foi preparado nas mesmas condições, apenas acrescentando $1 \mathrm{~g}$ de nióbia $\left(\mathrm{Nb}_{2} \mathrm{O}_{5}\right)$ ao béquer contendo os sais de ferro. Após a formação, os materiais foram lavados com $\mathrm{H}_{2} \mathrm{O}$ destilada até a neutralização e secos em estufa por $24 \mathrm{~h}$, a $60{ }^{\circ} \mathrm{C}$.

\section{Caracterização dos materiais}

Os materiais foram caracterizados por difratometria de raios- $\mathrm{X}$ (DRX), utilizando-se difratômetro da Philips, com variação angular de $20-80^{\circ}(2 \theta)$, radiação de $\operatorname{CoK} \alpha(\lambda=1,78897 \AA)$ e velocidade de exposição de $1^{\circ} 2 \theta \mathrm{min}^{-1}$, espectroscopia no infravermelho (trans- 
mitância), utilizando-se um espectrômetro Digilab Excalibur, série FTS 3000 e espectroscopia Mössbauer, CMTE modelo MA250, com fonte gama de ${ }^{57} \mathrm{Co} / \mathrm{Rh}$.

\section{Oxidação de cafeína e de catecol}

Os testes de oxidação foram realizados com soluções padrão de cafeína $25 \mathrm{mg} \mathrm{L}^{-1}$ e catecol 50 e $25 \mathrm{mg} \mathrm{L}^{-1}$, concentrações estas comparáveis às encontradas no efluente. Para cada processo de oxidação foram realizados testes com a solução contendo os contaminantes e o catalisador, para se avaliar a capacidade de adsorção desse material (testemunha). A oxidação foi monitorada por espectroscopia de UV-visível (Shimadzu-UV-1601 PC) no comprimento de onda característico da cafeína (274 nm) e do catecol (275nm).

\section{Produtos de oxidação da cafeína e catecol}

Os produtos de degradação da cafeína e do catecol, pelo processo Foto-Fenton foram analisados por LC/MS (ESI-MS Trap Agilent-1100). As amostras ionizadas foram inseridas no aparelho por infusão a um fluxo de $5 \mu \mathrm{L} \mathrm{min}^{-1}$, com controle de carga no quadropolo (ICC) ajustado para 30.000. A pressão para formação do spray foi de 0,8 bar.

A amostra de cafeína foi ionizada com tampão formato de amônio $\mathrm{pH} 5,5$ e analisada com temperatura do gás de secagem $\left(\mathrm{N}_{2}\right)$ de $325^{\circ} \mathrm{C}$ e fluxo de $4 \mathrm{~L} \mathrm{~min}^{-1}$, com potencial de extração de íons de $-3500 \mathrm{~V}$.

A amostra de catecol foi ionizada com tampão de hidróxido de amônio pH 10 e analisada com temperatura e fluxo do gás de secagem $\left(\mathrm{N}_{2}\right)$ de $200{ }^{\circ} \mathrm{C}$ e $6 \mathrm{~L} \mathrm{~min}^{-1}$, respectivamente, com potencial de extração de íons de $+3.500 \mathrm{~V}$.

\section{Sistema Fenton heterogêneo e Foto-Fenton}

Os testes de oxidação pelo sistema Fenton heterogêneo $\left(\mathrm{H}_{2} \mathrm{O}_{2}\right.$ + catalisador) foram realizados a $25{ }^{\circ} \mathrm{C}$, utilizando-se $100 \mathrm{~mL}$ de soluções padrão de cafeína e de catecol; $30 \mathrm{mg}$ do catalisador e 0,1 $\mathrm{mL}$ de $\mathrm{H}_{2} \mathrm{O}_{2}(30 \%)$. Essas condições foram escolhidas com base em trabalhos prévios do grupo. A oxidação por foto-Fenton (hv $+\mathrm{H}_{2} \mathrm{O}_{2}$ + catalisador) foi realizada nas mesmas condições, porém a reação foi conduzida sob exposição de uma lâmpada de mercúrio, com radiação em $254 \mathrm{~nm}$, mantida a uma distância de $16 \mathrm{~cm}$ do sistema catalítico.

\section{Sistema $\mathrm{UV} / \mathrm{H}_{2} \mathrm{O}_{2}$}

Os testes de oxidação por $\mathrm{UV} / \mathrm{H}_{2} \mathrm{O}_{2}$ foram realizados em condições ambientes, utilizando-se $100 \mathrm{~mL}$ de soluções padrão de cafeína e de catecol, $0,1 \mathrm{~mL}$ de $\mathrm{H}_{2} \mathrm{O}_{2}(30 \%)$, sob agitação constante e sob exposição de uma lâmpada de mercúrio com radiação em $254 \mathrm{~nm}$ a uma distância do sistema catalítico de $15 \mathrm{~cm}$.

\section{RESULTADOS E DISCUSSÃO}

\section{Caracterização dos materiais}

\section{Espectroscopia no infravermelho (FTIR)}

Os espectros dos materiais na região do infravermelho são mostrados na Figura 1.

Pela espectroscopia na região do infravermelho (FTIR), pôde-se confirmar a formação da goethita $(\alpha-\mathrm{FeOOH})$, pelos sinais característicos em $3.142 \mathrm{~cm}^{-1}$ de estiramento $\mathrm{OH}, 610$ ligação Fe-O e 888 e $790 \mathrm{~cm}^{-1}$, vibrações $\mathrm{OH}$ no plano e fora do plano. A separação dessas duas bandas foi de $98 \mathrm{~cm}^{-1}$, que pode ser indicativa de formação de um material com boa cristalinidade. ${ }^{9}$

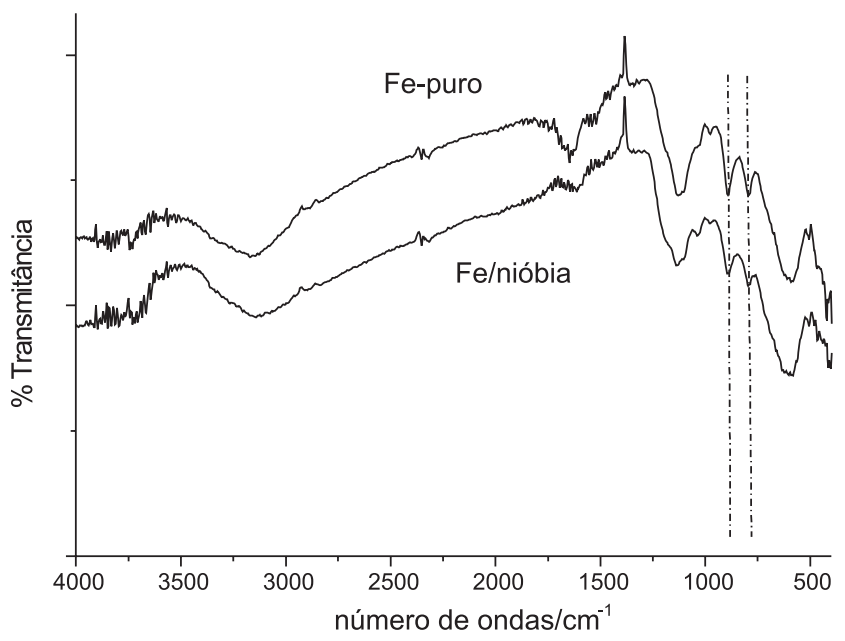

Figura 1. Espectroscopia de infravermelho dos materiais: Fe/Nb (óxido de ferro/oxido de nióbia) e Fe-puro (óxido de ferro)

Difratometria de raios- $X$

Os difratogramas de raios-X (DRX) do Fe-puro e do compósito $\mathrm{Fe} / \mathrm{Nb}$ são mostrados na Figura 2.

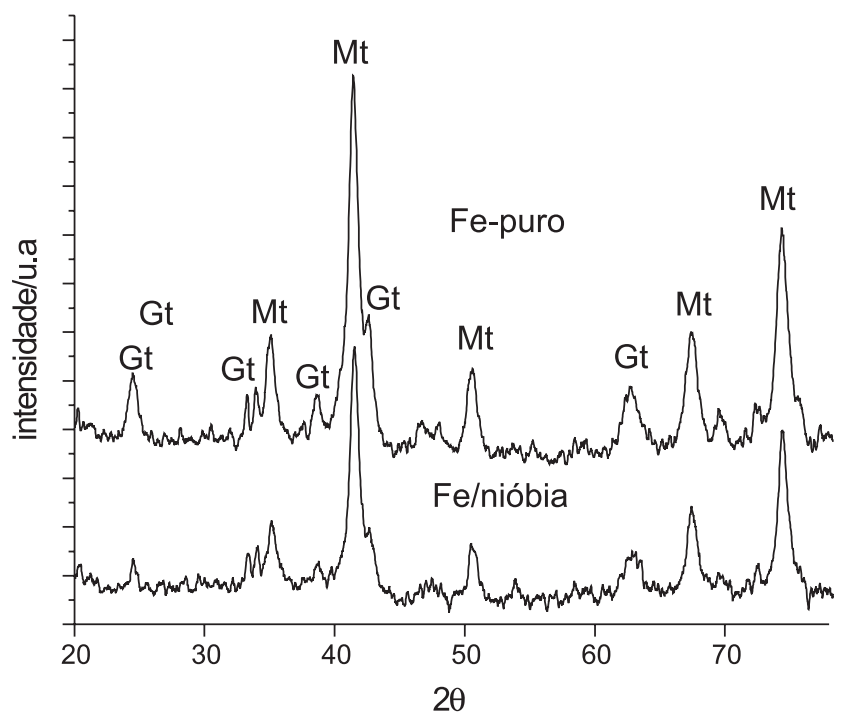

Figura 2. Difratograma de raios- $X$ dos materiais: $\mathrm{Fe} / \mathrm{Nb}$ (óxido de ferro/ óxido de nióbia) e Fe-puro (óxido de ferro)

Pelo difratograma de raios- $X$, pode-se observar a formação das fases ferruginosas goethita $(\alpha-\mathrm{FeOOH})$, evidenciada pelo espaçamentos basais em $\mathrm{d}=0,4193 ; 0,2098$ e $0,1613 \mathrm{~nm}$ e maghemita $\left(\gamma-\mathrm{Fe}_{2} \mathrm{O}_{3}\right)$ em $\mathrm{d}=0,2506$ e 0,2956 nm. ${ }^{9}$ Não foram observadas reflexões referentes à nióbia $\left(\mathrm{Nb}_{2} \mathrm{O}_{5}\right)$, sugerindo um possível caráter amorfo do material.

\section{Espectroscopia Mössbauer}

Os espectros Mössbauer dos materiais são mostrados na Figura 3 e os parâmetros Mössbauer estão listados na Tabela 1. Os espectros foram ajustados teoricamente considerando-se dois dupletos e dois sextetos.

Os dupletos A e B apresentam parâmetros hiperfinos característicos de $\mathrm{Fe}^{3+}$ de alto spin, provavelmente devidos à relaxação superparamagnética de óxidos com pequeno tamanho de partículas, ou dispersos em uma superfície.

Os sextetos A e B apresentam deslocamentos isoméricos $(\delta)$ e quadrupolar $(\varepsilon)$ abaixo dos valores característicos para a goethita e maghemita, possivelmente por efeito de pequenas partículas, com pa- 


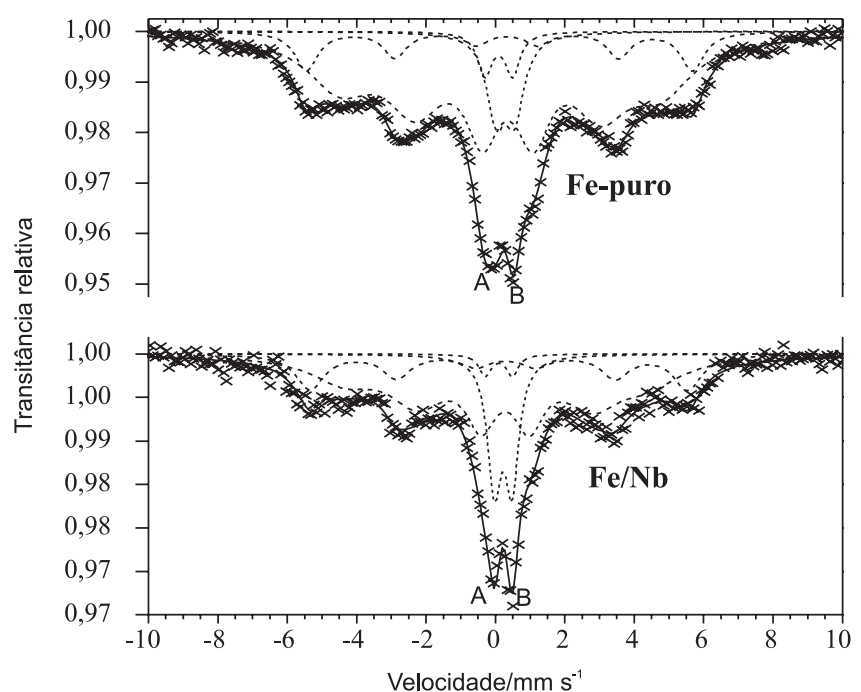

Figura 3. Espectros Mössbauer dos materiais a $298 \mathrm{~K}$ : Fe/Nb (óxido de ferro/ oxido de nióbia) e Fe-puro (óxido de ferro)

Tabela 1. Parâmetros Mössbauer dos espectros obtidos

\begin{tabular}{|c|c|c|c|c|}
\hline \multicolumn{2}{|c|}{ Amostra } & $\delta / \mathrm{mm} \mathrm{s}^{-1}$ & $\Delta, \varepsilon / \mathrm{mm} \mathrm{s}^{-1}$ & $\mathrm{~B}_{\mathrm{h} f} / \mathrm{T}$ \\
\hline \multirow{4}{*}{ Fe-puro } & Dupleto A & 0,34 & 0,80 & \\
\hline & Dupleto B & 0,43 & 0,54 & \\
\hline & Sexteto A & 0,36 & $-0,22$ & 34,5 \\
\hline & Sexteto B & 0,39 & $-0,21$ & 28,7 \\
\hline \multirow{4}{*}{$\mathrm{Fe} / \mathrm{Nb}$} & Dupleto A & 0,24 & 0,78 & \\
\hline & Dupleto B & 0,37 & 0,51 & \\
\hline & Sexteto A & 0,34 & $-0,20$ & 35,1 \\
\hline & Sexteto B & 0,28 & $-0,27$ & 30,2 \\
\hline
\end{tabular}

$\Delta=$ deslocamento isomérico, relativo ao $\alpha ; \Delta=$ desdobramento quadrupolar; $\varepsilon=$ deslocamento quadrupolar e $\mathrm{B}_{\mathrm{hf}}=$ campo magnético hiperfino.

drões espectrais de linhas alargadas. ${ }^{10}$ Porém, devido à magnetização observada no material e dos dados de difratograma de raios-X, onde apresenta espaçamento característico de fase cúbica, uma das fases de ferro presente pode ser maghemita.

\section{Oxidação do catecol}

A oxidação do catecol foi monitorada por espectroscopia de UV-visível, em $275 \mathrm{~nm}$. A porcentagem de remoção do composto é mostrada na Figura 4.

Dos resultados obtidos verifica-se que o catecol apresenta estabilidade à radiação $h v$. Os sistemas $\mathrm{H}_{2} \mathrm{O}_{2}+\mathrm{Fe} / \mathrm{Nb}$ e $h v+\mathrm{H}_{2} \mathrm{O}_{2}+$ $\mathrm{Fe} / \mathrm{Nb}$ demonstraram maior eficiência na oxidação, removendo $94 \mathrm{e}$ $95 \%$, respectivamente, do catecol presente na solução. O sistema $h v$ $+\mathrm{H}_{2} \mathrm{O}_{2}$ apresenta baixa atividade inicial, mas aos 240 min de reação removeu $80 \%$ do catecol.

A oxidação utilizando Fe-puro como catalisador não apresentou atividade na remoção do catecol. A melhor eficiência do catalisador $\mathrm{Fe} / \mathrm{Nb}$ pode ser devida à maior dispersão do óxido de ferro na superfície da nióbia. No caso do processo $h v+\mathrm{H}_{2} \mathrm{O}_{2}+\mathrm{Fe} / \mathrm{Nb}$, a radiação UV apenas acelera a reação inicial, o que pode ser devido à rápida formação de radicais hidroxila.

Os produtos de oxidação do catecol pelo sistema $h v+\mathrm{H}_{2} \mathrm{O}_{2}+$ $\mathrm{Fe} / \mathrm{Nb}$, foi monitorado por espectrometria de massas. Os resultados obtidos são apresentados na Figura 5.

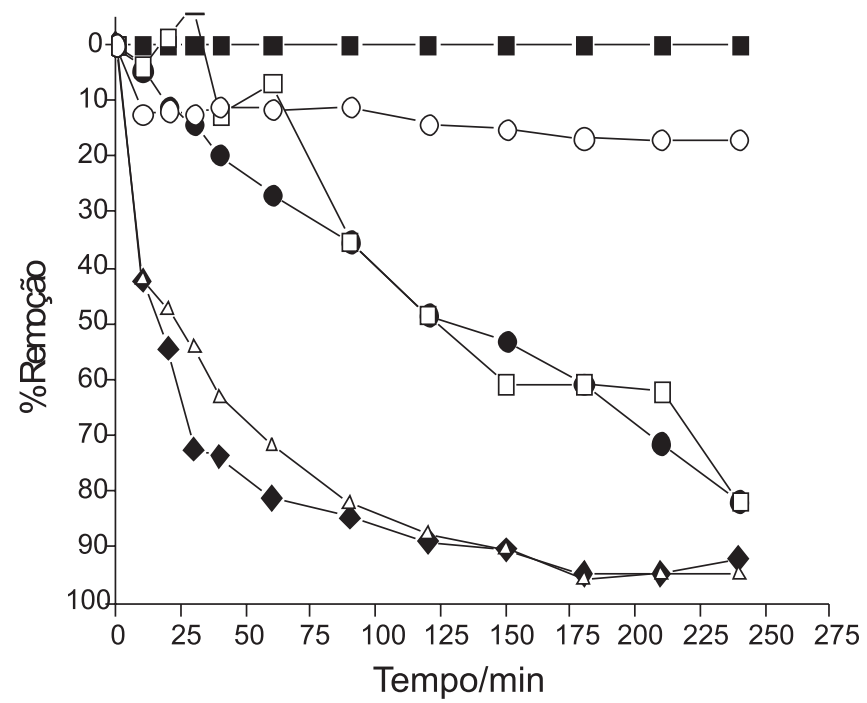

Figura 4. Porcentagem de remoção do catecol, utilizando os diferentes sistemas de oxidação com os materiais $\mathrm{Fe} / \mathrm{Nb}$ (óxido de ferro/óxido de nióbia) e Fe-puro (óxido de ferro) como catalisador $\left([]_{\text {inicial }}=5,6 \mathrm{mmol} \mathrm{L}^{-1}\right) . \mathbf{\square}=h v$, $\mathrm{O}=\mathrm{H}_{2} \mathrm{O}_{2}+\mathrm{Fe}$-puro, $\square=\mathrm{H}_{2} \mathrm{O}_{2}+\mathrm{Fe}$-puro $+h v, \bullet=\mathrm{H}_{2} \mathrm{O}_{2}+h v, \Delta=\mathrm{H}_{2} \mathrm{O}_{2}$ $+\mathrm{Fe} / \mathrm{Nb} \bullet \mathrm{H}_{2} \mathrm{O}_{2}+\mathrm{Fe} / \mathrm{Nb}+\mathrm{hv}$
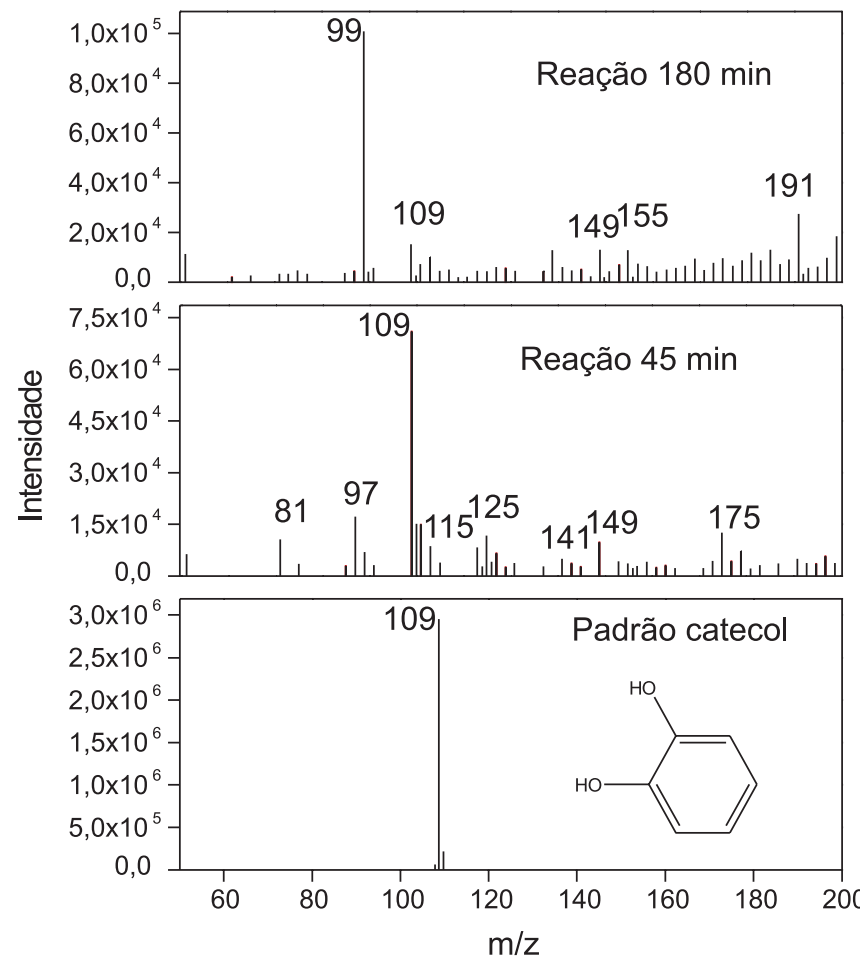

Figura 5. Monitoramento de oxidação do catecol por espectrometria de massas, utilizando o sistema $h v+H^{2} O^{2}+\mathrm{Fe} / \mathrm{Nb}(\mathrm{MS}$-)

Análises por electrospray mostram que, após 45 min de reação, o sinal $\mathrm{m} / \mathrm{z}=109$ referente ao catecol ainda é intenso, mas ocorre a formação de vários compostos intermediários. A evidência do processo de oxidação pode ser vista através da primeira e segunda hidroxilação com formação dos sinais $m / z=125$ e 141 . No entanto, após 180 min de reação, o sinal do catecol desaparece quase completamente, mas ainda se observam sinais de compostos intermediários, como o $m / z=99$. Os sinais $m / z$ observados encontram-se de acordo com o mecanismo proposto na literatura, tendo como produto final a formação do ácido 4-oxi-but-2-enóico, com massa molar $100(m / z=99),{ }^{11}$ como pode ser visto no Esquema 1. 


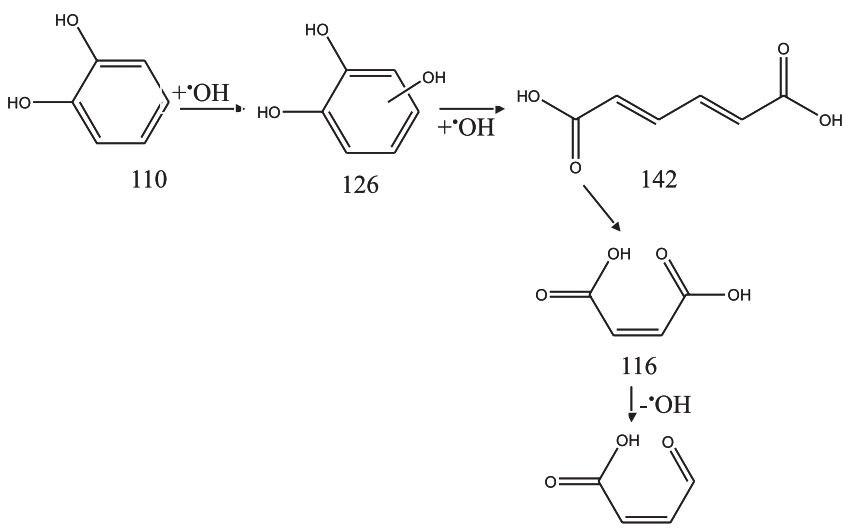

100

Esquema 1. Esquema de oxidação do catecol

\section{Oxidação da cafeína}

Na Figura 6 é mostrada a cinética de remoção da cafeína, monitorada por espectrometria de UV-visível em $274 \mathrm{~nm}$.

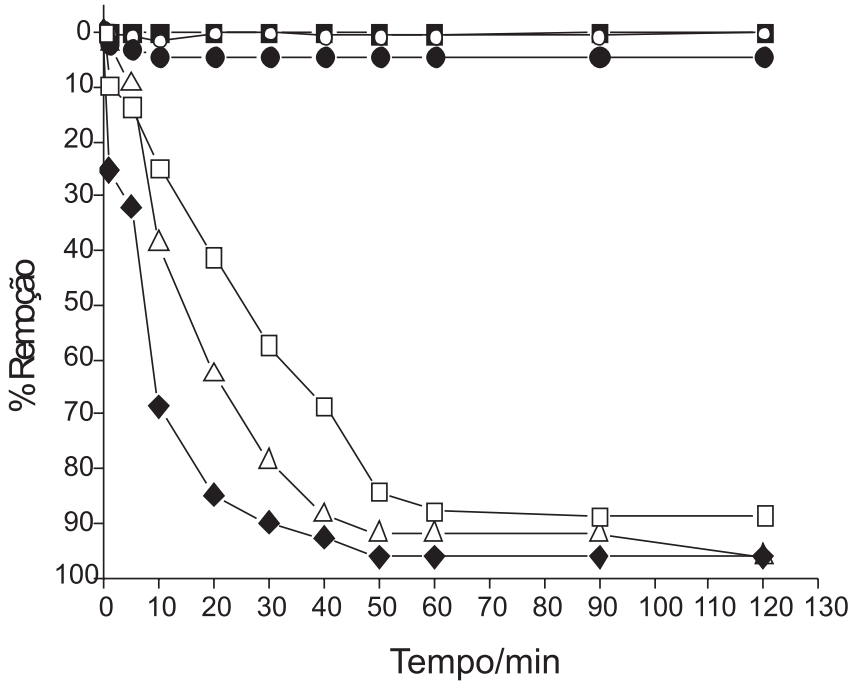

Figura 6. Cinética de remoção da cafeína utilizando os diferentes sistemas de oxidação e dos materiais Fe/Nb (óxido de ferro/óxido de nióbia) e Fe-puro (óxido de ferro) como catalisador ([ $]_{\text {inicial }}=7,76 \mathrm{mmol} \mathrm{L} \mathrm{L}^{1}$ ). $\mathbf{\square}=h \mathrm{v}, \bullet=\mathrm{H}_{2} \mathrm{O}_{2}$ + Fe-puro, $\mathrm{O}=\mathrm{H}_{2} \mathrm{O}_{2}+\mathrm{Fe} / \mathrm{Nb}, \square=\mathrm{H}_{2} \mathrm{O}_{2}+\mathrm{Fe}$-puro $+h v, \Delta=\mathrm{H}_{2} \mathrm{O}_{2}+h v$, $\bullet=\mathrm{H}_{2} \mathrm{O}_{2}+\mathrm{Fe} / \mathrm{Nb}+\mathrm{hv}$

Observa-se que a cafeína não é afetada pela radiação $h v$. Os sistemas $\mathrm{H}_{2} \mathrm{O}_{2}+\mathrm{Fe} / \mathrm{Nb}$ e $\mathrm{H}_{2} \mathrm{O}_{2}+\mathrm{Fe}$-puro não apresentaram atividade na remoção da cafeína, o que pode ser atribuído ao tipo de radical formado inicialmente. As fases ferruginosas formadas no material utilizado como catalisador foram goethita e maghemita (identificada pelo difratograma de raios-X), que contém somente $\mathrm{Fe}^{+3}$, responsável pela formação inicial de radicais (Equação 1).

$\mathrm{Fe}^{3+}+\mathrm{H}_{2} \mathrm{O}_{2}+h v \rightarrow \mathrm{Fe}(\mathrm{OOH})^{2+}+\mathrm{H}^{+} \rightarrow \mathrm{Fe}^{2+}+{ }^{\cdot} \mathrm{OOH}+\mathrm{H}^{+}$

O radical hidroperóxido ('OOH) formado pode degradar os orgânicos presentes $\left({ }^{\circ} \mathrm{OOH}+h v+\right.$ composto orgânico $\rightarrow$ composto orgânico oxidado), mas essa oxidação pode ser lenta ou, dependendo da estabilidade, o composto pode não ser oxidado devido ao baixo potencial do ${ }^{\circ} \mathrm{OOH}(1,25 \mathrm{~V})$. O radical ${ }^{\circ} \mathrm{OOH}$ em presença de outra molécula de $\mathrm{H}_{2} \mathrm{O}_{2}$ e radiação UV poderá formar o radical • $\mathrm{OH}$ (Equação 2).
$\mathrm{H}_{2} \mathrm{O}_{2}+{ }^{\cdot} \mathrm{OOH}+h v \rightarrow \cdot \mathrm{OH}+\mathrm{H}_{2} \mathrm{O}+\mathrm{O}_{2}$

Esse radical ( $\left.{ }^{\circ} \mathrm{OH}\right)$ é considerado um oxidante forte, com potencial padrão de $2,06 \mathrm{~V}$.

O processo de fotólise do peróxido de hidrogênio, $\left(\mathrm{H}_{2} \mathrm{O}_{2}+h v \rightarrow 2\right.$ $\cdot \mathrm{OH})$, apresentou boa eficiência na remoção da cafeína. Esse processo apresenta eficiência devido à formação de dois radicais hidroxilas, altamente oxidantes, capazes de oxidar compostos simples, mesmo mais estáveis que a cafeína. ${ }^{12-14}$

Para o sistema $\mathrm{H}_{2} \mathrm{O}_{2}+h v+\mathrm{Fe} / \mathrm{Nb}$, observa-se rápida degradação da cafeína: aos 20 min de reação mais de $85 \%$ da cafeína já haviam sido degradados, atingindo um máximo de degradação aos 50 min de reação. $\mathrm{O}$ aumento de velocidade pode ser decorrente de um efeito sinérgico entre os sistemas $\mathrm{H}_{2} \mathrm{O}_{2}+h v$ e $\mathrm{H}_{2} \mathrm{O}_{2}+\mathrm{Fe} / \mathrm{Nb}$, pois o sistema sem radiação $\left(\mathrm{H}_{2} \mathrm{O}_{2}+\mathrm{Fe} / \mathrm{Nb}\right)$ mostrou ser inativo para a degradação. Para o processo $h v+\mathrm{H}_{2} \mathrm{O}_{2}+$ Fe-puro verifica-se decréscimo da eficiência do sistema, quando se compara com o $h v+\mathrm{H}_{2} \mathrm{O}_{2}$, sugerindo que o catalisador (Fe-puro) pode estar dispersando ou absorvendo a radiação incidida.

Na Figura 7, são apresentados os resultados do monitoramento da oxidação da cafeína pelo sistema $\mathrm{H}_{2} \mathrm{O}_{2}+h v+\mathrm{Fe} / \mathrm{Nb}$, realizado por espectrometria de massas.

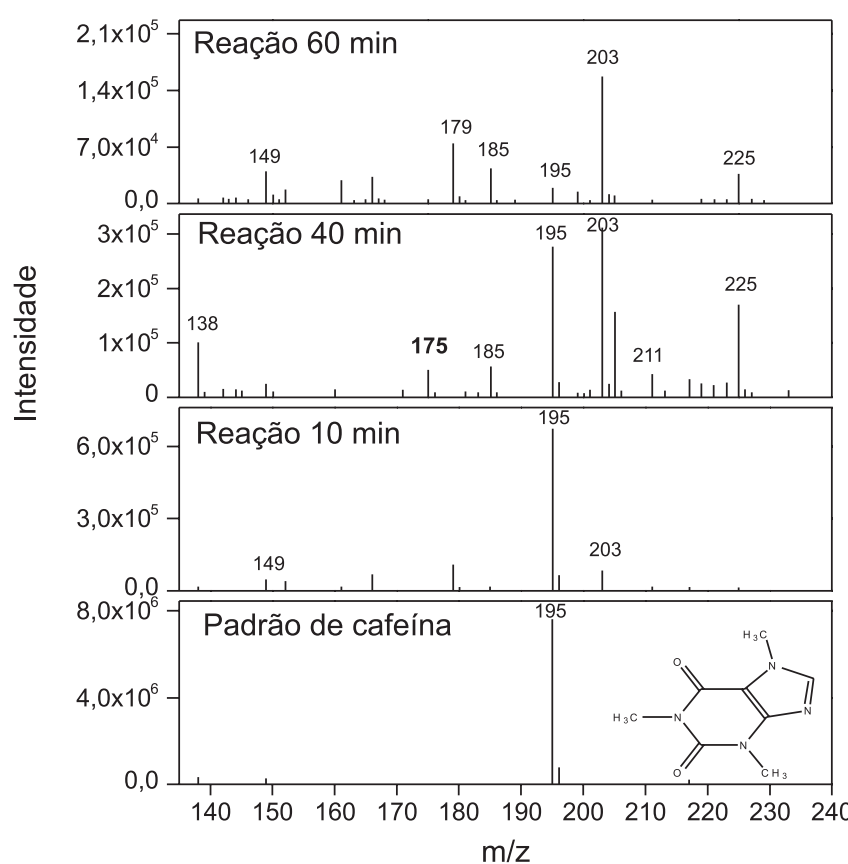

Figura 7. Monitoramento de oxidação da cafeína por espectrometria de massas, utilizando o sistema $\mathrm{H}_{2} \mathrm{O}_{2}+h v+\mathrm{Fe} / \mathrm{Nb}(\mathrm{MS}+)$

Verifica-se diminuição da intensidade do sinal $m / z=195(\mathbf{M}+\mathrm{H})$, o que evidencia a oxidação da cafeína, simultaneamente à formação de outros sinais $\mathrm{m} / \mathrm{z}$, devido a produtos do processo de oxidação. $\mathrm{O}$ sinal em $m / z, 203(202+\mathrm{H})$ pode ser evidência do processo de mineralização da cafeína (três hidroxilações, seguido pela descarboxilação).

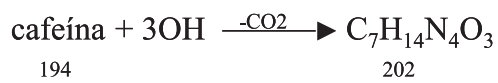

$\mathrm{O}$ sinal em $m / z=138$ é atribuído à perda do fragmento $\mathrm{H}_{3} \mathrm{CNCO}$, da molécula de cafeína. O sinal $m / z=175(174+\mathrm{H})$, aos $40 \mathrm{~min}$ de reação, é proposto como um rearranjo da molécula, segundo o esquema apresentado no Esquema $2 .^{15}$ 


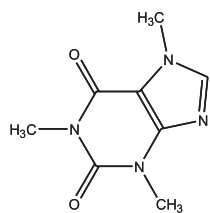

194

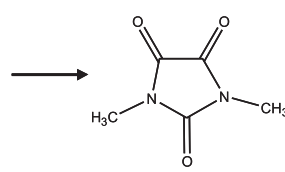

142

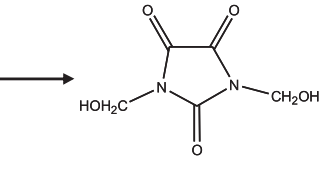

174
Esquema 2. Esquema de formação do sinal m/z 175

Os resultados obtidos neste trabalho sugerem ser o radical hidroxila $\left({ }^{\circ} \mathrm{OH}\right)$ o principal agente oxidante do material orgânico. Porém, a presença de radicais ${ }^{\circ} \mathrm{OOH}$ é responsável pelo aumento significativo da velocidade da reação, sob a radiação UV, na medida em que são gerados pelo catalisador $\mathrm{Fe} / \mathrm{Nb}$ e transformados em radicais ${ }^{\circ} \mathrm{OH} .{ }^{15-17}$ $\mathrm{O}$ material Fe-puro não apresentou nenhuma atividade para os compostos testados. Isso pode ser devido à boa cristalinidade observada pelo DRX e também à menor superfície de contato deste, em relação ao compósito.

\section{CONCLUSÃO}

O catalisador Fe/nióbia mostrou eficiência na degradação dos compostos testados, sob radiação ultra-violeta.

Os testes de oxidação monitorados por espectrometria de massas (ESI) sugerem hidroxilações sucessivas das moléculas, seguidas de perda de $\mathrm{CO}_{2}$; a completa mineralização do composto pode ocorrer com maior tempo de reação. Outros testes serão realizados, para se verificar o tempo de mineralização dos contaminantes e também no efluente da despolpa, que contém substâncias de outros grupos químicos.

\section{REFERÊNCIAS}

1. Gonçalves, M.; Dissertação de Mestrado, Universidade Federal de Lavras, Brasil, 2006.

2. Matos, A. T.; Fukunaga, D. C.; Pinto, A. B.; Russo J. R.; Resumos do simpósio de pesquisa dos cafés do Brasil, Vitória, Brasil, 2001.

3. Freire, R. S.; Pelegrini, R.; Kubota, I. T.; Durán, N.; Peralta-Zamora, P.; Quim. Nova 2000, 23, 504.

4. Kunz, A.; Peralta-Zamora, P.; Moraes, S. G.; Duran, N.; Quim. Nova 2002, 25, 78 .

5. Dunford, B. H.; Coord. Chem. Rev. 2002, 233, 311.

6. Pera-Titus, M.; García-Molina, V.; Baños, M. A.; Gimenez, J.; Esplugas, S.; Appl. Catal., B 2004, 47, 219.

7. Yang, G. P.; Zhao, X. K.; Sun, X. J.; Lu, X. L.; J. Hazard. Mater. 2005, 126, 112.

8. Oliveira, L. C. A.; Silva, C. N.; Yoshida, M. I.; Lago, R. M.; Carbon 2004, 42, 2279.

9. Cornell, R. M.; Schwertmann, U.; The Iron Oxides, $3^{\text {rd }}$ ed., Weinheim-VHC: New York, 2003.

10. Oliveira, L. C. A.; Gonçalves, M.; Guerreiro, M. C.; Fabris, J. D; Pereira, M. C.; Sapag, K.; Appl. Catal., A 2007, 2, 117.

11. Santos, A.; Yustos, P.; Quintanilla, A.; Rodríguez, S.; García-Ochoa, F.; Appl. Catal., B 2002, 39, 97.

12. Oliveira, L. C. A.; Gonçalves, M.; Fabris, J. D.; Guerreiro, M. C.; Resumos do $13^{\circ}$ Congresso Brasileiro de Catálise $/ 3^{\circ}$ Congresso de Catálise do Mercosul, Foz do Iguaçu, Brasil, 2005.

13. Schrank, S. G.; José, H. J.; Moreira, R. F. P. M.; Schröder, H. Fr.; Chemosphere 2005, 60, 644.

14. Tiburtius, E. R. L.; Peralta-Zamora, P.; Emmel, A. E. S. L.; Quim. Nova 2005, 28, 61 .

15. Dalmázio, I.; Santos, L. S.; Lopes, R. P.; Eberlin, M. N.; Augusti, R.; Environ. Sci. Technol. 2005, 39, 5982.

16. Benitaz, F. J.; Real, F. J.; Acero, J. L.; Leal, A. I. E.; Garcia, C.; J. Hazard. Mater. 2005, 126, 31.

17. Trovó, A. G.; Villa, R. D.; Nogueira, R. F. P.; Quim. Nova 2005, 28, 847. 